\section{Helicobacter pylori - Prävalenz in der deutschen Bevölkerung}

\begin{abstract}
Zusammenfassung: Im Rahmen des Bundes-Gesundheitssurveys wurde eine Immunglobulin-G-Antikörperbestimmung gegen das Bakterium Helicobacter pylori im Serum der Probanden durchgeführt. Für die deutsche Bevölkerung wurde eine Gesamtprävalenz von $40 \%$ ermittelt. Geschlechtsunterschiede wurden nicht festgestellt. Ein Vergleich zwischen den Surveyteilnehmern aus den alten und neuen Bundesländern ergab eine erhöhte Prävalenzrate in allen Altersgruppen bei den östlichen Probanden. Mögliche Ursachen und Einflussfaktoren werden diskutiert.
\end{abstract}

Helicobacter Pylori - Prevelance in the German Population: Within the framework of the German National Health Interview and Examination Survey determinations of IgG-antibodies against the bacterium Helicobacter pylori in serum samples of study participants were conducted. The prevalence for the total population of Germany was $40 \%$. No differences could be found between sexes. The comparison between study participants from the old and those from the new (territory of the former GDR) federal states of Germany did show that the prevalence rates were significantly higher in all agegroups of the eastern part (former GDR) of Germany. Possible causes and factors of influence are discussed.

Key words: Helicobacter Pylori-Antibodies - German National Health Interview and Examination Survey - Prevalence

\section{Einführung}

Seit es den Australiern Marshall und Warren [1] gelang, das Bakterium Helicobacter pylori zu kultivieren, befassen sich zahlreiche Wissenschaftler in aller Welt mit diesem Keim, untersuchen seine Herkunft, Struktur, die Transmissionswege, die Prävalenz in Abhängigkeit der verschiedensten Faktoren, seine Bedeutung für die verschiedensten Magenerkrankungen.

So war man bis zu den 80er Jahren der Auffassung, dass die Gastritis nur eine Gewebeveränderung sei, die durch lebenslange Belastung der Magenschleimhaut mit Stoffen wie Alkohol, Nikotin, Kaffee und Medikamente entsteht. Stress, Hektik

Gesundheitswesen 2000; 62: 598-603

(C) Georg Thieme Verlag Stuttgart · New York ISSN 0941-3790

\author{
C. Seher, W. Thierfelder, R. Dortschy \\ Robert Koch-Institut, Berlin
}

und ungesunde Ernährung wurden für die Entwicklung eines Magengeschwürs verantwortlich gemacht.

Heute wissen wir, dass die früheren Lehrmeinungen über die Gastritis und die Ulkus-Krankheiten in Magen und Zwölffingerdarm unzureichend waren und dass die Theorien über die Entstehungsmechanismen des Magenkarzinoms ergänzt werden müssen. So werden heute 95\% der Zwölffingerdarmgeschwüre und 70\% der Magengeschwüre als Folge einer Helicobacter-pylori-Infektion interpretiert. Die Erhöhung des Magenkarzinomrisikos durch die Besiedelung der Magenschleimhaut mit Helicobacter pylori wird allgemein mit einem Faktor zwischen 3 und 6 angegeben [2-5].

Obwohl es bereits vielfältige Studien zur Prävalenz von Helicobacter-pylori-Infektionen in den verschiedensten Ländern und Regionen gibt, die ein starkes Gefälle von 96\% in Algerien bis zu 15\% bei weißen Australiern aufzeigen, fehlen weltweit umfangreiche Untersuchungen, die einen genauen Überblick über den Durchseuchungsgrad eines Landes geben können.

Diese Möglichkeit ist jetzt für Deutschland mit der Durchführung des Bundes-Gesundheitssurveys gegeben. Mit einer derartigen Aussage für die Gesamtbevölkerung lassen sich gesundheitspolitische Strategien entwickeln, die ihre Schwerpunkte sowohl in der Infektionsprävention als auch in der Verhinderung von Folgekrankheiten (Gastritis, Ulkus, Karzinom) sehen sollten.

\section{Material und Methoden}

Im Rahmen des Bundes-Gesundheitssurveys 1997/98 wurde an 6748 Personen im Alter von 18 bis 79 Jahren (Gesamtstichprobe: 7124) eine Antikörperbestimmung gegen Helicobacter pylori durchgeführt (zu Studienpopulation und Stichprobenauswahl siehe Bundes-Gesundheitssurvey [6]).

Es kam der ELISA-Test (enzyme-linked immunosorbent assay) der Firma Hoffmann La Roche zur Anwendung. Dies ist ein Zweischritt-Assay zur Bestimmung von IgG-Antikörpern gegen Helicobacter pylori im Serum. Die Messung erfolgte am Analysenautomaten Cobas Core der gleichen Firma. Der Variationskoeffizient (VK) der mitgeführten Kontrolle lag zwischen 3,5 und 7\%. Alle Angaben zur Präanalytik sind unter [6] ausführlich beschrieben.

Es wurden die Altersgruppen 18-29 Jahre, 30-39 Jahre, 40-49 Jahre, 50-59 Jahre, 60-69 Jahre und 70-79 Jahre gebildet. Mit 
Tab. 1 Helicobacter-pylori-Antikörper in Deutschland (gewichtet)

\begin{tabular}{lllllll}
\hline $\begin{array}{l}\text { Helicobacter-pylori-Antikörper } \\
\text { positiv }\end{array}$ & Altersgruppen & & & \\
\hline
\end{tabular}

Ausnahme der höchsten Altersgruppe waren alle Gruppen beiderlei Geschlechts annähernd gleich verteilt.

Zum Rauchverhalten wurden 3 Kategorien gebildet: Habe nie geraucht, rauche selten bzw. habe früher geraucht und rauche täglich.

Die ärztliche Befragung hinsichtlich aufgetretener Magenerkrankungen wurde folgendermaßen durchgeführt:

- Haben oder hatten Sie jemals eine Magenschleimhautentzündung (Gastritis): ja oder nein?

- Haben oder hatten Sie jemals ein Magen- bzw. Zwölffingerdarmgeschwür: ja oder nein?

Aufgrund der zu erwartenden Ost-West-Unterschiede zum Gesundheitszustand wurde im Survey ein disproportionaler Ansatz der Stichprobe zugunsten der neuen Bundesländer gewählt. Um aber mit den Daten des Bundes-Gesundheitssurveys (1997/98) eine aktuelle Aussage für die gesamte Bevölkerung der Bundesrepublik Deutschland treffen zu können, wurde im Survey ein entsprechender Wichtungsfaktor eingeführt [7]. Im Gegensatz zu den Prävalenzberechnungen wurden die statistischen Tests mit ungewichteten Daten durchgeführt.

Das Merkmal „soziale Schicht“, das zur Beurteilung der Schichtspezifik (Unter-, Mittel- und Oberschicht) verwendet wurde, ist entsprechend der DAE-Empfehlung (Deutsche Arbeitsgemeinschaft Epidemiologie) aus Einkommen, Bildung und beruflicher Position zusammengesetzt [8].

Die Variable „Beginn Kitabesuch: 0-2 Jahre“ schließt den Besuch einer Krippeneinrichtung bis zum Alter $<3$ Jahre ein.

Das Merkmal „Anzahl Geschwister“ wurde in 4 Kategorien unterteilt: Keine Geschwister (0), ein Geschwisterkind (1), zwei Geschwister (2), drei und mehr Geschwister (3).

Die statistische Auswertung erfolgte mittels SPSS Programmpaket Version 9.01.

Die relativen Risiken wurden mittels Odds Ratios (OR) geschätzt, welche bekanntermaßen mit „Bias“ behaftet sind. Bei kleinen Prävalenzen (<10\%) sind diese vernachlässigbar, wäh- rend bei größeren zu berücksichtigen ist, dass der Odds Ratio die Risiken überschätzt.

\section{Ergebnisse}

Eine einfache Häufigkeitsbetrachtung brachte folgende $\mathrm{Er}$ gebnisse: Für $40 \%$ aller untersuchten Probanden $(n=6748)$ wurden Antikörper gegen Helicobacter pylori nachgewiesen. Ein geschlechtsspezifischer Unterschied konnte weder insgesamt, noch innerhalb der einzelnen Altersgruppen festgestellt werden (Tab. 1).

Im Ost-West-Vergleich treten deutlich sichtbare Differenzen in allen Gruppen zutage (Tab. 1). Generell weisen die Probanden aus den alten Bundesländern (West) eine geringere Helicobacter-pylori-Prävalenz gegenüber denen aus den neuen Bundesländern (Ost) auf. Dies kann für alle Altersgruppen bestätigt werden. Die höchste Differenz liegt mit 17,3\% bei den 40- bis 49-Jährigen, dann verringern sich die Unterschiede mit dem Alter kontinuierlich bis auf $8,1 \%$. In der jüngsten Altersgruppe sind die Differenzen mit 4,6\% am niedrigsten. Der deutliche Anstieg der Antikörperträger mit dem Alter ist in Ost und West analog.

Tab. 2 zeigt die Probanden mit positiven bzw. negativen Helicobacter-pylori-Antikörperbefunden in Abhängigkeit von der sozialen Schicht [9]. Sowohl in Ost als auch in West weisen die Untersuchten, die der Unterschicht zugeordnet wurden, eine deutlich höhere Prävalenzrate im Vergleich zu den höheren sozialen Schichten auf. Im Osten beträgt die Differenz der Unter- zur Oberschicht 13,5\%, im Westen sogar 23,4\%. Interessant ist, dass es in den neuen Bundesländern zwischen Mittel- und Oberschicht keinen nennenswerten Unterschied bezüglich der Infektionsrate gibt $(0,9 \%)$, während er im Westen stark ausgeprägt ist (12\%).

Der Besuch einer Kinderkrippe bzw. Kindertagesstätte der Probanden bis zu einem Alter von 3 Jahren wurde erfasst. $15,5 \%$ aller Surveyteilnehmer aus den östlichen Bundesländern haben eine Kinderkrippe besucht (in den alten Bundesländern waren es nur 2,5\%). Davon wiederum waren rund $36 \%$ im Osten und ca. 47\% im Westen Antikörperträger gegen Helicobacter pylori.

\begin{tabular}{lllll}
\hline $\begin{array}{l}\text { Helicobacter-pylori-Antikörper } \\
\text { positiv }\end{array}$ & soziale Schicht & & gesamt \\
\hline & & & & \\
Ost & Unterschicht & Mittelschicht & Oberschicht & \\
West & $57,9 \%$ & $45,3 \%$ & $44,4 \%$ & $48,2 \%$ \\
gesamt & $49,0 \%$ & $37,6 \%$ & $25,6 \%$ & $37,5 \%$ \\
\hline
\end{tabular}

Tab. 2 Helicobachter-pylori-Antikörper in Abhängigkeit von der sozialen Schicht (gewichtet) 
Den Einfluss der Geschwisterzahl auf die Infektionsrate lässt Abb. 1 erkennen. Bei allen Probanden steigt die Prävalenz mit zunehmender Geschwisterzahl, wobei generell die Teilnehmer aus den neuen Bundesländern eine um mindestens 10\% höhere Rate aufweisen.

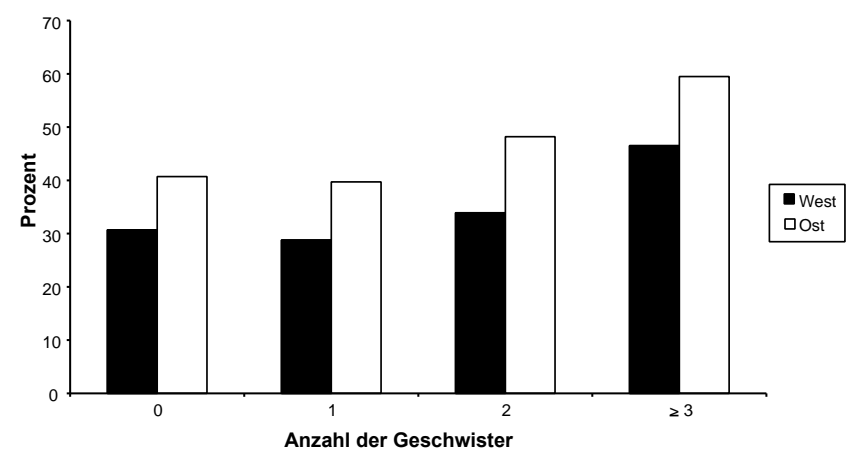

Abb. 1 Prävalenz von Helicobacter-pylori-Antikörpern in Abhängigkeit von der Anzahl der Geschwister

Der Zusammenhang zwischen positivem Helicobacter-pyloriAntikörper und Gastritis bzw. Ulkus zeigt sich in Tab.3. Hier sind die erwarteten Häufigkeiten bei fehlendem Einfluss der Antikörper auf die entsprechenden Magenerkrankungen den tatsächlich gefundenen gegenübergestellt.

1567 von den 6748 Probanden gaben bei der ärztlichen Befragung an, dass sie gegenwärtig oder zu einem früheren Zeitpunkt eine Gastritis haben oder hatten. Bei 46\% dieser Probanden wurde ein positiver Antikörpernachweis erbracht.

Von den 529 Untersuchten, die ein Magen- oder Zwölffingerdarmgeschwür angegeben hatten, wiesen $61 \%$ einen positiven Antikörper gegen Helicobacter pylori auf.

Die Chi²-Tests ergaben für beide Variablen einen hochsignifikanten Zusammenhang mit dem Nachweis von Helicobacterpylori-Antikörpern.

Ein Einfluss des Rauchens auf die Prävalenzrate der Helicobacter-pylori-Infektion wurde bei dieser eindimensionalen Betrachtungsweise nicht festgestellt ( $\mathrm{Chi}^{2}$-Test nicht signifikant).

Die Berücksichtigung mehrerer gleichzeitig wirkender Einflüsse erfordert die Anwendung multivariater Modelle. $\mathrm{Zu}$ nächst wurde zur Abschätzung des Risikos, eine Helicobacterpylori-Infektion zu bekommen, eine logistische Regression mit den Variablen Geschlecht, Ost/West, Alter, soziale Schicht und Rauchgewohnheiten durchgeführt. Dabei wurden paar- weise Interaktionseffekte zwischen diesen Variablen berücksichtigt.

In Tab. 4 sind diese Ergebnisse zusammengestellt. Ein Geschlechtsunterschied war nicht nachweisbar. Die Bevölkerung in den neuen Bundesländern hat ein höheres Risiko, eine Helicobacter-pylori-Infektion zu bekommen als die Bevölkerung in den westlichen Bundesländern $(\mathrm{OR}=2,2)$. Das Risiko ist für die Unterschicht (Gesamtdeutschland) um das 2,9fache und das der Mittelschicht um das 1,8fache höher als das der Oberschicht. Rauchen hat in diesem Modell keinen signifikanten Einfluss auf eine Infektion.

Tab. 4 Relative Risiken für die Helicobacter-pylori-Infektion

\begin{tabular}{lll}
\hline & OR & $\mathrm{P}$ \\
Geschlecht (männlich) & 1,00 & \\
Geschlecht (weiblich) & 1,08 & 0,62 \\
Bundesländer (Ost) & 1,00 & \\
Bundesländer (West) & 0,46 & $<0,0001$ \\
Alter (18-29 Jahre) & 1,00 & \\
Alter (30-39 Jahre) & 1,96 & $<0,0001$ \\
Alter (40-49 Jahre) & 3,28 & $<0,0001$ \\
Alter (50-59 Jahre) & 4,25 & $<0,0001$ \\
Alter (60-69 Jahre) & 6,32 & $<0,0001$ \\
Alter (70-79 Jahre) & 6,96 & $<0,0001$ \\
Schichtindex (Unterschicht) & 2,88 & $<0,0001$ \\
Schichtindex (Mittelschicht) & 1,77 & $<0,01$ \\
Schichtindex (Oberschicht) & 1,00 & \\
Rauchen (nie) & 1,00 & \\
Rauchen (gelegentlich o. früher) & 1,03 & 0,91 \\
Rauchen (täglich) & 1,18 & 0,39
\end{tabular}

In einem zweiten Schritt wurde, wiederum in einem logistischen Regressionsmodell, der Einfluss der Helicobacter-pylori-Infektion auf Ulkus und Gastritis untersucht. Geschlecht, Ost/West, Alter, Schichtindex und Rauchen wurden als Kovariaten in dieses Modell mit aufgenommen. Die Ergebnisse sind in Tab. 5 dargestellt.

Der Einfluss einer Helicobacter-pylori-Infektion auf das Entstehen eines Magen- bzw. Zwölffingerdarmgeschwürs wird durch ein OR von 1,8 ersichtlich.

Das Risiko, an einem Ulkus zu erkranken, steigt konstant mit dem Alter an und wird auch durch das Rauchen maßgeblich beeinflusst $(\mathrm{OR}=2,2)$. Eine Abhängigkeit von der Schichtzugehörigkeit ist erkennbar; so ist das Risiko, einen Ulkus zu bekommen, für die Probanden der Unter- und Mittelschicht um das 1,5fache höher im Vergleich zu denen der Oberschicht.

Der Einfluss der Variablen auf das Entstehen einer Gastritis ist generell bedeutend schwächer. So kann nur eine geringfügige Abhängigkeit zwischen angegebener Magenschleimhautentzündung und Antikörperträgern registriert werden $(\mathrm{OR}=1,2)$.
Helicobacter-pylori-Antikörper (\%)

\begin{tabular}{llllll}
\hline & & erwartet & & ermittelt & \\
\multirow{3}{*}{ Gastritis } & positiv & negativ & positiv & negativ \\
& ja & 41,1 & 58,9 & 46,2 & 53,8 \\
\multirow{3}{*}{ Ulkus } & nein & 41,1 & 58,9 & 39,6 & 60,4 \\
& ja & 41,1 & 58,9 & 61,2 & 38,8 \\
& nein & 41,1 & 58,9 & 39,4 & 60,6 \\
\hline
\end{tabular}

Tab. 3 Zusammenhang von Helicobacterpylori-Antikörpern mit Angaben zu Magenerkrankungen 


\begin{tabular}{|c|c|c|c|c|}
\hline & \multicolumn{2}{|l|}{ Ulkus } & \multicolumn{2}{|c|}{ Gastritis } \\
\hline & OR & $\mathrm{p}$ & OR & $\mathrm{p}$ \\
\hline Geschlecht (männlich) & 1,00 & & 1,00 & \\
\hline Geschlecht (weiblich) & 0,73 & $<0,01$ & 1,38 & $<0,0001$ \\
\hline Bundesländer (Ost) & 1,00 & & 1,00 & \\
\hline Bundesländer (West) & 1,19 & 0,09 & 1,29 & 0,0001 \\
\hline Alter (18-29 Jahre) & 1,00 & & 1,00 & \\
\hline Alter (30-39 Jahre) & 2,59 & $<0,001$ & 1,82 & $<0,0001$ \\
\hline Alter (40-49 Jahre) & 4,88 & $<0,0001$ & 2,22 & $<0,0001$ \\
\hline Alter (50-59 Jahre) & 9,69 & $<0,0001$ & 2,92 & $<0,0001$ \\
\hline Alter (60-69 Jahre) & 9,96 & $<0,0001$ & 3,16 & $<0,0001$ \\
\hline Alter (70-79 Jahre) & 11,34 & $<0,0001$ & 2,60 & $<0,0001$ \\
\hline Schichtindex (Unterschicht) & 1,46 & $<0,05$ & 0,77 & $<0,01$ \\
\hline Schichtindex (Mittelschicht) & 1,46 & $<0,01$ & 0,87 & 0,0610 \\
\hline Schichtindex (Oberschicht) & 1,00 & & 1,00 & \\
\hline Rauchen (nie) & 1,00 & & 1,00 & \\
\hline Rauchen (gelegentlich o. früher) & 1,63 & $<0,0001$ & 1,52 & $<0,0001$ \\
\hline Rauchen (täglich) & 2,25 & $<0,0001$ & 1,47 & $<0,0001$ \\
\hline Helicobacter-pylori-Ak (positiv) & 1,79 & $<0,0001$ & 1,18 & $<0,01$ \\
\hline
\end{tabular}

Tab. 5 Untersuchte Einflussgrößen auf Ulkus und Gastritis

\section{Diskussion und Schlussfolgerungen}

Die durchschnittliche Prävalenz von Probanden mit positivem Antikörper gegen Helicobacter pylori ist $40 \%$ und entspricht damit dem europäischen Mittelwert [10,21]. Ein Unterschied zwischen den Geschlechtern wurde nicht festgestellt. Dies steht weitgehend in Übereinstimmung mit der Literatur, allerdings gibt es auch Autoren [11], die eine höhere Prävalenz bei Männern registrierten.

Eine Abhängigkeit der Infektionsrate vom Alter scheint offensichtlich (Tab.1). Der Anstieg erfolgt kontinuierlich von 21,7\% in der jüngsten Gruppe bis auf 60,5\% bei den 70-79-Jährigen. Diese Kontinuität ist für Ost und West analog. Da eigentlich auch alle anderen nationalen und internationalen Studien [12-15] einen Anstieg der Helicobacter-pylori-Infektion mit dem Alter beobachten, könnte der Schluss nahe liegen, dass die Ansteckungsrate unter älteren Menschen besonders hoch ist. Dies erscheint jedoch unlogisch. Angesichts von Untersuchungen, die belegen, dass eine Infektion wahrscheinlich bereits in der frühen Kindheit $[14,16,17]$ erfolgt (und unbehandelt auch bestehen bleibt), muss der kontinuierliche Anstieg als Kohortenphänomen interpretiert werden. Dies bedeutet, dass die Infektionsrate der heute 50 - oder 60 -Jährigen in ihrer Kindheit höher lag als es bei den jetzt 20- oder 30-Jährigen der Fall war. Festgestellt hat man dies überwiegend dadurch, dass Probandenkollektive, die über 10 Jahre lang beobachtet wurden $[13,18]$, nur geringfügige Neuinfektionen aufwiesen (durchschnittlich 0,3\% pro Jahr). So wurden 600 Teilnehmer des Spandauer Gesundheitstests (Freiwilligenuntersuchung im Berliner Bezirk Spandau), die zwischen 1982 und 1995 mindestens fünfmal im Abstand von 2 Jahren auf Helicobacter-pylori-Antikörper untersucht worden sind, zur Berechnung der Neuinfektionsrate herangezogen [19]. Über die Hälfte dieser Probanden wies ein negatives Testergebnis über den gesamten Beobachtungszeitraum auf. Diese wurden zu denen mit einer erfolgten Neuinfektion (8 Teilnehmer) in Beziehung gesetzt, daraus ergab sich eine jährliche Infektionsrate von nur $0,2 \%$.

Bekannt ist die Abhängigkeit der Infektionsrate vom sozioökonomischen Status. Die Aussagen in der Literatur beziehen sich auf die verschiedensten Parameter, wie hygienische Bedingungen (Vorhandensein eines Badezimmers, einer Toilette etc. in der Wohnung), Bildung und Einkommen [20]. In der vorliegenden Studie wurde das Merkmal „soziale Schicht“ gebildet, das sich aus Einkommen, Bildung und beruflicher Position zusammensetzt und eine Unterteilung in 3 Schichten: Unter-, Mittel- und Oberschicht ermöglicht. Bei gesamtdeutscher Betrachtung zeigt sich, dass die Unterschicht mit 50,9\% Antikörperträgern weit über den beiden höheren Schichten (39,3 bzw. 28,9\%) liegt. Eine umfangreiche europäische Studie, an der sich 17 Populationen beteiligten [21], kam zu ähnlichen Ergebnissen. So betrug hierbei die Infektionsrate der Personen mit hoher Bildung 34\% im Vergleich zu 62\% derer, die nur eine niedrige Ausbildung erfahren hatten. Einen analogen Effekt stellten die Autoren auch in Abhängigkeit vom Familieneinkommen fest. Zwischen den Probanden aus den neuen (Ost) und den alten Bundesländern (West) sind statistisch gesicherte Differenzen in allen 3 Schichten nachweisbar. Die Infektionsrate der Probanden „Ost“ ist generell höher als die der Probanden „West“ (Tab. 2). Die größten Unterschiede liegen mit $18,8 \%$ in der Oberschicht, dann folgt die Unterschicht mit 8,9\%, die Differenz der Probanden aus den Mittelschichten beträgt dagegen nur 7,7\%. Die Ergebnisse der logistischen Regression unterstützen und präzisieren diese Aussage: Die Bevölkerung in den neuen Bundesländern hat somit ein 2,2fach höheres Risiko, eine Helicobacter-pylori-Infektion zu bekommen als die Bevölkerung in den westlichen Bundesländern. Das ermittelte Risiko für die Unterschicht (Ost und West) liegt um den Faktor 2,9 über dem der Oberschicht (Tab.4). Da, wie bereits oben ausgeführt, die Infektion überwiegend in der Kindheit erfolgt und meistens ohne Therapie lebenslang erhalten bleibt, werden auch diese Ost-West-Unterschiede unabhängig vom Zeitpunkt der Vereinigung Deutschlands noch lange sichtbar sein. Hygienische Bedingungen und die Ernährungsweise, die in die „soziale Schicht“ aufgrund von Einkommen und Bildung einfließen, sind sicher als Hauptursachen für die gefundenen Differenzen anzusehen. Der erwartete Einfluss des Kinderkrippenbesuches der Probanden aus den östlichen Ländern auf die Infektionsrate konnte nicht bestätigt werden. Von diesen 1364 untersuchten Surveyteilnehmern gaben nur 15,5\% (alte Bundesländer: 2,5\%) an, eine Kinderkrippe besucht zu haben. Dies korreliert 
nicht mit den Angaben in den Statistischen Jahrbüchern der DDR - dort variieren die Zahlen zwischen 9,1\% (1955) und 72,7\% (1985). Die prozentuale Verteilung der HelicobacterAntikörperträger innerhalb der Krippen- bzw. Kitabesucher liegt dagegen bei $36 \%$ im Osten und $47 \%$ im Westen. Allerdings sind die geringen Fallzahlen nicht repräsentativ, um Rückschlüsse auf die Gesamtbevölkerung anstellen zu können. Auch der Einfluss der Geschwisterzahl trägt nicht zur Klärung der Unterschiede zwischen den Prävalenzen in Ost und West bei. Denn während in den alten Bundesländern der Anteil der Probanden mit 3 und mehr Geschwistern fast 10\% über dem in den neuen Ländern liegt (1 oder 2 Geschwister bilden keinen wesentlichen Unterschied zwischen Ost und West), ist die Antikörper-Prävalenzrate im Westen deutlich niedriger (Abb. 1). Die Prävalenz der H.p.-positiven Probanden ist generell im Osten höher, die Differenzen liegen in Abhängigkeit von der Anzahl der Geschwister zwischen 11 und 14\%.

Die Helicobacter-pylori-induzierte Gastritis ist mit 80 bis $90 \%$ aller Gastritiden die häufigste Gastritis weltweit [22]. 1567 Probanden gaben im Rahmen der ärztlichen Befragung an, eine Gastritis zu haben, aber nur bei rund $46 \%$ wurden auch Helicobacter-pylori-Antikörper ermittelt (Tab.3). Dieses Ergebnis muss nicht im Widerspruch zu obiger Aussage stehen, weil eine Probandenaussage ohne klinische Befundung gerade bei dieser Krankheit nicht eindeutig sein muss. So wird z.B. bei einer diagnostizierten atrophischen Gastritis, insbesondere bei älteren Patienten, häufig keine Besiedelung der Magenschleimhaut mit Helicobacter pylori mehr beobachtet [23-25]. Die Wissenschaftler erklären dies mit dem Verschwinden der Keime aus atrophischen und metaplastischen Bereichen, weil diese in einem solchen Milieu auf längere Zeit nicht persistieren können.

Der Zusammenhang zwischen einer Helicobacter-pylori-Infektion und einem angegebenen Ulkus ist mit 61\% zwar eindeutiger als bei der Gastritis (Tab.3), liegt aber doch deutlich unter den Literaturwerten. Aber auch hier muss die fehlende klinische Diagnostik, wenn auch sicher im geringeren Maße, berücksichtigt werden. Die durchgeführte logistische Regression mit der Zielstellung der Risikoabschätzung, eine Gastritis bzw. eine Ulkus-Krankheit zu bekommen, lässt erkennen, dass in diesem Modell die Einflussgrößen Alter und Rauchen einen höheren Stellenwert als die Helicobacter-pylori-Infektion besitzen (Tab. 5).

Der Faktor Rauchen hat bei der eindimensionalen Betrachtungsweise keinen Einfluss auf die Besiedelung der Magenschleimhaut mit Helicobacter pylori. Dies korreliert auch mit den meisten Literaturangaben. Da die Infektion überwiegend in der Kindheit erfolgt, ist das eigene Rauchverhalten aus Altersgründen wenig relevant. Interessanter wäre die Betrachtung der Rauchgewohnheiten im Umfeld des damaligen Kindes (Eltern, Geschwister). Dieser Gedanke ist dann allerdings auch für die „soziale Schicht“ umzusetzen, denn die Schichtzugehörigkeit zwischen Kindheit und Erwachsenenalter kann durchaus differieren.

Nach dem heutigen Wissensstand liegt in den industrialisierten Ländern die jährliche Reinfektionsquote nur zwischen 0,3 und $2 \%$. Diese Tatsache wäre ein Grund, eine Eradikationstherapie an Kindern und Jugendlichen bei positivem Helicobacter-pylori-Befund durchzuführen, um einer Entwicklung der
H.p.-Folgekrankheiten wie Gastritis, Ulkus und Karzinom vorzubeugen. In dem vom Robert-Koch-Institut geplanten Kinder- und Jugendsurvey sollen auch die Helicobacter-pyloriAntikörperträger im Alter von 0 bis 18 Jahren ermittelt werden. Die geplante Stichprobe von 20000 Probanden wird eine repräsentative Aussage über den Durchseuchungsgrad bei Kindern und Jugendlichen in der Bundesrepublik Deutschland ermöglichen und somit einen wesentlichen Beitrag zur Therapiefrage (national und international) liefern können.

Der Bundesgesundheitssurvey ermittelte bei 40\% der Probanden positive Antikörper gegen Helicobacter pylori. Dieses Ergebnis lässt allerdings keine Schlussfolgerungen hinsichtlich einer vorliegenden akuten oder chronischen Infektion $\mathrm{zu}$, auch eine überstandene Infektion (Immunisierung) ist nicht auszuschließen. Deshalb müsste jetzt eigentlich bei diesen Antikörperträgern klinisch (endoskopisch) überprüft werden, ob die Notwendigkeit einer Therapie gegeben ist.

Da jedes Jahr etwa 1 Million Deutsche an einer Helicobacterpylori-Gastritis und deren Folgen erkranken und die Behandlungskosten auf ca. 4 Milliarden DM geschätzt werden [25] nach neueren Berechnungen ermöglichen die Eradikationstherapien eine Einsparung von 3,2 bzw. 3,8 Milliarden DM $[26,27]-$, sollte auch der ökonomische Aspekt bei der Entscheidung über eine derartige Prophylaxe in Betracht gezogen werden.

\section{Literatur}

${ }^{1}$ Marshall B, Warren JR. Unidentified curved bacilli on gastric epithelium in active chronic gastritis. Lancet 1983; 1: 1273-1275

2 Forman D, Webb P, Parsonnet J. Helicobacter pylori and gastric cancer. Lancet 1994; 343: 243-244

3 Talley NJ, Zinsmeister AR, Weaver A. Gastric adenocarcinoma and Helicobacter pylori infection. J Natl Cancer Inst 1991; 93: 1734-1739

${ }^{4}$ Sipponen P, Kosunen TU, Valle J, Riihela M, Seppala K. Helicobacter pylori infection and chronic gastritis in gastric cancer. J Clin Pathol 1992; 45: 319-323

${ }^{5}$ Hansson L, Engstrand L, Nyren $\mathrm{O}$ et al. Helicobacter pylori infection independent risk indicator of gastric adenocarcinoma. Gastroenterology 1993; 105: 1098-1103

${ }^{6}$ Der Bundesgesundheitssurvey 1997/98. Das Gesundheitswesen 1998, Sonderheft, 2: S59-S114

7 Thefeld W, Stolzenberg H, Bellach BM. Bundes-Gesundheitssurvey: Response, Zusammensetzung der Teilnehmer und Non-Responder-Analyse. Das Gesundheitswesen, Sonderheft, 1999; 2: S57-S61

8 Winkler J. Die Messung des sozialen Status mit Hilfe eines Index in den Gesundheitssurveys der DHP. Ahrens W, Bellach BM, Jöckel KH (Hrsg). RKI-Schriften, 1998: 69-74

${ }^{9}$ Ahrens W, Bellach BM, Jöckel KH. Messung soziodemographischer Merkmale in der Epidemiologie. RKI-Schriften, 1998; 1

${ }^{10}$ Megraud F, Brassens-Robbe MP, Denis F, Belbouri A, Hoa DQ. Seroepidemiology of Campylobacter pylori infection in various populations. J Clin Microbiol 1989; 27: 1870-1873

11 Graham DY, Malaty HM, Evans DG, Evans DJ, Klein PD, Adam E. Epidemiology of Helicobacter pylori in an asymptomatic population in the United States: Effect of age, race and socioeconomic status. Gastroenterology 1991; 100: 1495-1501

12 Banatvala N, Mayo K, Megraud F et al. The cohort effect and Helicobacter pylori. J Infect Dis 1993; 168: 219-221

${ }^{13}$ Cullen DJ, Collins BJ, Christiansen KJ et al. When is Helicobacter pylori infection acquired?. Gut 1994; 34: 1681-1682 
${ }^{14}$ Lindkvist P, Asrat D, Nilsson I et al. Age at acquisition of Helicobacter pylori infection: comparison of a high and low prevalence country. Scand J Infect Dis 1996; 28: 181-184

15 Forbes GM, Glaser ME, Cullen DJ et al. Duodenal ulcer treated with Helicobacter pylori eradication: seven-year follow-up. Lancet 1994; 343: 258-260

16 O'Donohoe JM, Sullivan PB, Scott R et al. Recurrent abdominal pain and Helicobacter pylori in a community-based sample of London children. Acta Pediatr 1996; 85: 961-964

17 Mitchell HM, Li YY, Hu PJ et al. Epidemiology of Helicobacter pylori in Southern China: identification of early childhood as the critical period for acquisition. J Infect Dis 1992; 166: 149-153

18 Kuipers EJ, Uyterlinde AM, Pena AS et al. Long-term sequelae of Helicobacter pylori gastritis. Lancet 1995; 345: 1525-1528

${ }^{19}$ Seher C, Thefeld W. Helicobacter pylori und Magenkrankheiten: derzeitiger Erkenntnisstand. Ernährungs-Umschau 1998; 45: 116-120

${ }^{20}$ Logan RPH, Gummett PA, Wallace P et al. The urban epidemiology of Helicobacter pylori. Hepato-Gastroenterol 1993; 40

21 The Eurogast-Study Group. Epidemiology of, and risk factors for, Helicobacter pylori infection among 3194 asymptomatic subjects in 17 populations. Gut, 1996; 34: 1672-1676

22 Stolte M. Pathologie der Helicobacter pylori-Krankheiten. Malfertheiner P (Hrsg). Helicobacter pylori- von der Grundlage zur Therapie, Stuttgart: Thieme, 1994: 19-37

${ }^{23}$ Karnes WE, Samloff IM, Siurala M et al. Positive serum antibody and negative tissue staining for Helicobacter pylori in subjects with atrophic body gastritis. Gastroenterol 1991; 101: 167-174

24 Shibata T, Imoto I, Ohuchi Y et al. Helicobacter pylori infection in patients with gastric carcinoma in biopsy and surgical resection specimens. Cancer 1996; 27: 1044-1049

25 Ärztliche Praxis, 1997; 49: 12

26 Oberender P. Medizinischer Standard und ökonomisches Einsparungspotential bei Ulcuserkrankungen. Der Niedergelassene Arzt 1996; 1: II-XII

27 Oberender P. Behandlungsmöglichkeiten des Helicobacter pylori: Kausaltherapie aus wirtschaftlicher Sicht. Leber Magen Darm 1997; 27: 30-34

\section{Charlotte Seher \\ Robert Koch-Institut \\ Postfach 650280 \\ 13302 Berlin}

\section{BUCHBESPRECHUNGEN}

Laer, Lutz von: Frakturen und Luxationen im Wachstumsalter. 3., neubearb. Auflage, Ersch.-J. 1996; XIII, 450 S.; Georg Thieme Verlag, Stuttgart; KST; DM 228,00/EUR 116,57/ ATS 1664,00 / CHF 202,00; ISBN 3-13-674303-2

Die dritte Auflage des Buches zeigt im Vergleich zu den beiden vorhergehenden Auflagen eine überschaubarere Gliederung. Jedem Kapitel ist jetzt eine Zusammenschau vorangestellt, die neben einem kurzen Schema der Verletzungsform orientierende Hinweise für die akute Behandlung und die Nachbehandlung gibt und auf spezielle Probleme hinweist. In den einzelnen Kapiteln finden sich dann umfassend die spezielle Diagnostik und Therapie. Insbesondere auf Fehler in der Therapie und daraus resultierenden Komplikationen, die im Kindesalter häufig von größerer Tragweite als im Erwachsenenalter sind, wird hingewiesen.

Ergänzt wird diese Auflage durch einen Anhang der Beckenfrakturen, Wirbelsäulenverletzungen und pathologischen Frakturen abhandelt. Im Vergleich zum Gesamtumfang des Buches erscheint dieser Anhang relativ kurz.
Das schon in den vorangehenden Auflagen umfangreiche Bildmaterial wurde erweitert und durch die Darstellung moderner Therapieverfahren ergänzt.

Das Buch ist ein Standardwerk für jeden in der Kinder- und Jugendlichen Traumatologie tätigen Arzt C. Beske, Kiel

Müller, Reinhold T./Karl Otto Bergmann: Haftungsverfahren und Risikomanagement in Orthopädie und Chirurgie. Auflage 2000; 159 S.; Georg Thieme Verlag, Stuttgart; Gebunden; DM 148,00/EUR 75,67/ATS 1080,00/CHF 131,00; ISBN 3-13-124561-1

Insbesondere in den operativen Fächern - hier Chirurgie und Orthopädie - nimmt die Bedeutung von Haftungsfragen einerseits durch die Zunahme der Stellung von Ansprüchen durch Patienten, andererseits aber auch durch die immer differenziertere Rechtsprechung immer stärker zu.

Das vorliegende Werk führt sowohl die medizinischen als auch die juristischen Gesichtspunkte bei den einzelnen Therapieverfahren zusammen. Neben den Kapiteln Einwilligung und Aufklärung des Patienten wird sowohl die Aufklärung als auch die Problematik der Fahrlässigkeit und Sorgfalt anhand von vielen in der Praxis täglich vorkommenden Fällen abgehandelt. Arbeitsrecht, Dokumentation sowie juristische Abläufe im Straf- und Zivilverfahren sind u.a. Inhalt der weiteren Kapitel.

Durch eine schematische Gliederung des Textes und eine prägnante Formulierung, ergänzt durch wichtige Textpassagen, wird das Werk sehr übersichtlich. Das Buch gibt jedem in der Chirurgie und Orthopädie tätigen Arzt wichtige Hinweise für dieses immer mehr in den Vordergrund tretende Gebiet. C. Beske, Kiel

Scharinger, Christian (Hrsg.). Perspektiven der Jugendgesundheit; Reihe Gesundheitswissenschaften. Bd. 12; Auflage 2000; 123 S.; Institut für Gesellschafts- und Sozialpolitik, Johannes-Kepler-Universität Linz; Broschiert; DM 20,00/ öS 132,00/sFr 20,00/EUR 9,60; ISBN 3-900581-27-4

Dieser Tagungsband der Jugendgesundheitskonferenz 1999 des Landes Oberösterreich schlägt mit insgesamt fünf Beiträgen einen Bogen von der Theorie und Empirie hin zur praktischen Umsetzung. Gemeinsames Grundverständnis ist, Jugendliche nicht „von außen“, d.h. als Problem- oder Risikogruppe zu betrachten, sondern stets die Perspektive der Jugendlichen und deren Ressourcen mit zu reflektieren. Das entspricht einer Selbstkonstituierung der Jugend als Population, wie sie bereits am Anfang der Geschichte der Jugend stand, wie W. Dür in seinem Beitrag „Schöner Vogel Jugend?" beschreibt. Empirische Ergebnisse aus Oberösterreich und Deutschland zeigen, dass sich zentrale Gesundheitsprobleme gewandelt haben und auch bei Jugendlichen chronische und psychosomatische Erkrankungen sowie emotionale Befindlichkeitsstörungen im Zentrum stehen. Hierbei weisen Unterschiede zwischen Mädchen und Jungen auf unterschiedliche personale und soziale Schutzfaktoren hin (P. Kolip). Diese zu stärken (Empowerment) sollte der Ansatz von Interventionen sein. Einen Eindruck von solchen häufig schon existierenden, jedoch in der Regel schlecht dokumentierten Projekten gibt ein Beitrag über ein Stuttgarter Projekt der gesundheitsbezogenen Jungenpädagogik.

T. Elkeles, Berlin 